\title{
Development of a Roadmap for the Implementation of a Sustainable Mobility Action Plan in University Campuses of Emerging Countries
}

\author{
María Teresa Tormo-Lancero ${ }^{1 *}$, Pedro Valero-Mora ${ }^{1}$, Jaime Sanmartin ${ }^{1}$, \\ Mar Sánchez-García ${ }^{1}$, Panagiotis Papantoniou ${ }^{2}$, George Yannis ${ }^{3}$, Francisco Alonso ${ }^{1}$ and \\ Eva Campos-Díaz ${ }^{1}$ \\ ${ }^{1}$ University Research Institute on Traffic and Road Safety, University of Valencia, Valencia, Spain, ${ }^{2}$ Department of Surveying \\ and Geoinformatics Engineering, University of West Attica, Athens, Greece, ${ }^{3}$ Department of Transportation Planning and \\ Engineering, National Technical University of Athens, Athens, Greece
}

OPEN ACCESS

Edited by:

Paolo Santi,

Massachusetts Institute of

Technology, United States

Reviewed by:

Mingming Liu,

Dublin City University, Ireland

Feybi Ariani Goni,

Brno University of

Technology, Czechia

*Correspondence:

María Teresa Tormo-Lancero

m.teresa.tormo@uv.es

Specialty section:

This article was submitted to

Urban Transportation Systems and

Mobility,

a section of the journal

Frontiers in Sustainable Cities

Received: 15 February 2021

Accepted: 07 December 2021

Published: 19 January 2022

Citation:

Tormo-Lancero MT, Valero-Mora $P$ Sanmartin J, Sánchez-García M, Papantoniou P, Yannis G, Alonso F and Campos-Díaz E (2022)

Development of a Roadmap for the Implementation of a Sustainable Mobility Action Plan in University

Campuses of Emerging Countries.

Front. Sustain. Cities 3:668185 doi: 10.3389/frsc.2021.668185
Universities are centers of knowledge and their Campuses are like small cities, thus making them the ideal place to develop, apply and evaluate policies and tools for innovative mobility solutions that can subsequently be extended to other contexts. A review of mobility measures in different European Universities has revealed that many of them apply policies to promote sustainable mobility, but there is a significant lack of standardized mobility plans and roadmaps for their successful implementation. The objective of the present work is to develop a successful roadmap, which is necessary for the smooth implementation of a mobility plan, as it has been found through a thorough review of good practices in Universities. Within this framework, a customizable standardized Roadmap design is proposed, which consists of two documents: a tactical document that provides a global and sequential vision of the entire plan, and an operational document that details the actions for each strategic line. This roadmap is accompanied by a catalog of objectives, measures, and cost and impact indicators. We consider this design instructive for universities because of its universal characteristics in Emerging Countries. To ensure this, it is necessary to apply this roadmap and carry out the corresponding evaluation.

Keywords: university campus, sustainable mobility plan, roadmap for decision makers, action plan, gap analysis, SWOT analysis, advances in emerging countries, sustainable mobility measures in universities

\section{INTRODUCTION}

Mobility is a social need that undoubtedly has a positive effect on the life of citizens; however, it also has several negative side effects on air quality, congestion, noise and accidents, thus making mobility a key priority for modern cities (European Union, 2011). To achieve this goal, the participation of different actors, good intradepartmental coordination between administrations, and the development of different lines of work are important. In this sense, universities play an important role in the fulfillment of the mobility objectives, since they are usually located inside city center and have a well-defined young population with a flexible mindset. Consequently, universities have the opportunity to play a significant role in promoting sustainable mobility in their environments. Besides, universities are knowledge nodes, which makes them the right place 
to test innovative solutions in terms of mobility, and to propose strategies on public transport, information and communication technology (ICT) and shared mobility (Papantoniou et al., 2017).

Many official sources, international organizations and researchers have reported how the global health crisis due to COVID-19 has affected mobility. Mobility restrictions and fear of contagion have indirectly encouraged private car, bicycle use and walking. The pandemic has also fueled negative attitudes toward public transportation and reduced its use in consequence (ETSC, 2020; Haas et al., 2020; Nieuwenhuijsen, 2020; OECD/ITF, 2020; Riggs, 2020; Thomas et al., 2021; Zhang et al., 2021). Taking advantage of positive attitudes toward cycling and walking, reestablishing confidence in public transport and discourage the excessive use of the private car should be top priorities of authorities in the close future.

Universities are an example that can be used to promote and transmit sustainable mobility habits to society, since today's students will have a relevant role in the society of the near future (Silva and Ferreira, 2008). In recent years, there has been a trend to move universities out of cities, based on the global trend of establishing new campuses or relocating them on the outskirts of the city or in rural areas, in order to place them away from congested urban centers. Over the years, they have turned into independent communities, reaching the size, infrastructures and activity levels of small cities (Tsirimpa et al., 2015), thus making them an excellent test bed for the application and evaluation of innovative mobility policies and tools. On the other hand, other universities, that are still within cities, generate a significant impact on them (Tolley, 1996; Balsas, 2003; Gamberi et al., 2015). Moreover, there are different types of public in universities, such as employees, students and visitors, all of them having different mobility habits and attitudes.

Sustainable Urban Mobility Plans (SUMP) ${ }^{1}$ have become an integrated planning tool, that is used in order to meet the mobility needs of cities and people, aiming at improving the urban mobility and accessibility of urban areas, as well as at providing high-quality services and sustainable modes of mobility and transport to, from and within the examined area according to their needs (Wefering et al., 2014; Torrisi et al., 2020; Khashaypoor et al., 2021). The development of roadmaps is specified in the 2011 European Union Transport Book as one of the instruments available to help meet these objectives, and it is part of a strategy aimed at developing costeffective interventions to improve quality, safety, integration and accessibility of public transport services. It also aims at raising awareness on sustainable transport options, such as public transport, carpooling, cycling and walking, as positive alternatives to the use of private transport.

Despite the potential usefulness of SUMPs and RoadMaps ${ }^{2}$, to the date there is still no review of sustainable mobility strategies in universities that has found standardized methods of development; on the contrary, the strategies used by universities vary widely. For example, some universities have implemented mobility plans based on the plans of the cities in which they were

\footnotetext{
${ }^{1}$ http://www.eltis.org/mobility-plans/european-platform

${ }^{2} \mathrm{http}: / /$ www.urban-transport-roadmaps.eu/
}

located, while others have implemented measures more specific to their conditions. This review has shown that most university mobility plans are limited to providing mobility solutions to and from the campus, but not within the campus (Tormo Lancero et al., 2018; Papantoniou et al., 2020). It is quite certain that we have not found any scientific contribution that provides a standard roadmap for mobility managers yet.

Because of this gap, it might be useful for Universities to have a standardized tool that allows for a successful implementation of the mobility plan, as well as a strategic vision of the plan, that guarantees the commitment of the decision-makers and that can be shared with other Universities as good practices. In short, a ready guide for decision makers that strengthens and supports a successful implementation of the Action Plan. The Action Plan proposed in the CAMP-sUmp project is shown in Figure 1 (Regattieri et al., 2018; Papantoniou et al., 2020).

The objective of this paper is to propose a standard roadmap that serves as a planning tool for the development of efficient and effective mobility strategies in the University context, ensuring transferability to University Campuses with different environmental characteristics and socio-economic conditions. The roadmap should be a supportive tool for the implementation of the action plan, and it should ensure the cooperation, dialogue, capacity and commitment of decision-makers in Sustainable Mobility Planning. A strategic vision must be established, that encompasses the steps to be followed so that each objective from different work lines can be achieved, as well as ensuring that the appropriate actions in the short, medium and long term are implemented, and that the tools and time needed in a hypothetical context are available, including the problems that may arise and the actors that should be involved in each case. Finally, it must be possible to evaluate the impact of the measures.

However, it must be taken into account that universities, despite sharing a number of common elements and characteristics, have a number of specificities that require different mobility objectives and measures. Therefore, it would be a mistake to design a Static Roadmap for all Universities. This position implies the need for a standard roadmap for all Universities, with clear elements and in the format of a fillable template that allows for a completion adapted to the objectives and measures of each University. Some authors, such as Gori et al. (2012) have stated that a classification in mobility solutions based on the specific characteristics of each country is required. In order to know how to design the roadmap, it is necessary to check if this hypothesis is true. According to the guidelines provided by Wefering et al. (2014), the first step to successfully develop a mobility plan is to conduct an assessment at the beginning, analyzing the baseline situation, needs, future challenges and priorities, identifying strengths and weaknesses of the current planning practices, and understanding the potential to successfully create a Sustainable Urban Mobility Plan. This assessment should determine how closely current practices align with the established activities, and it should identify barriers that could influence the plan development process (Wefering et al., 2014; Canitez, 2020; Foltnová et al., 2020).

Based on this premise, an evaluation of the scenarios in which higher education institutions find themselves could 


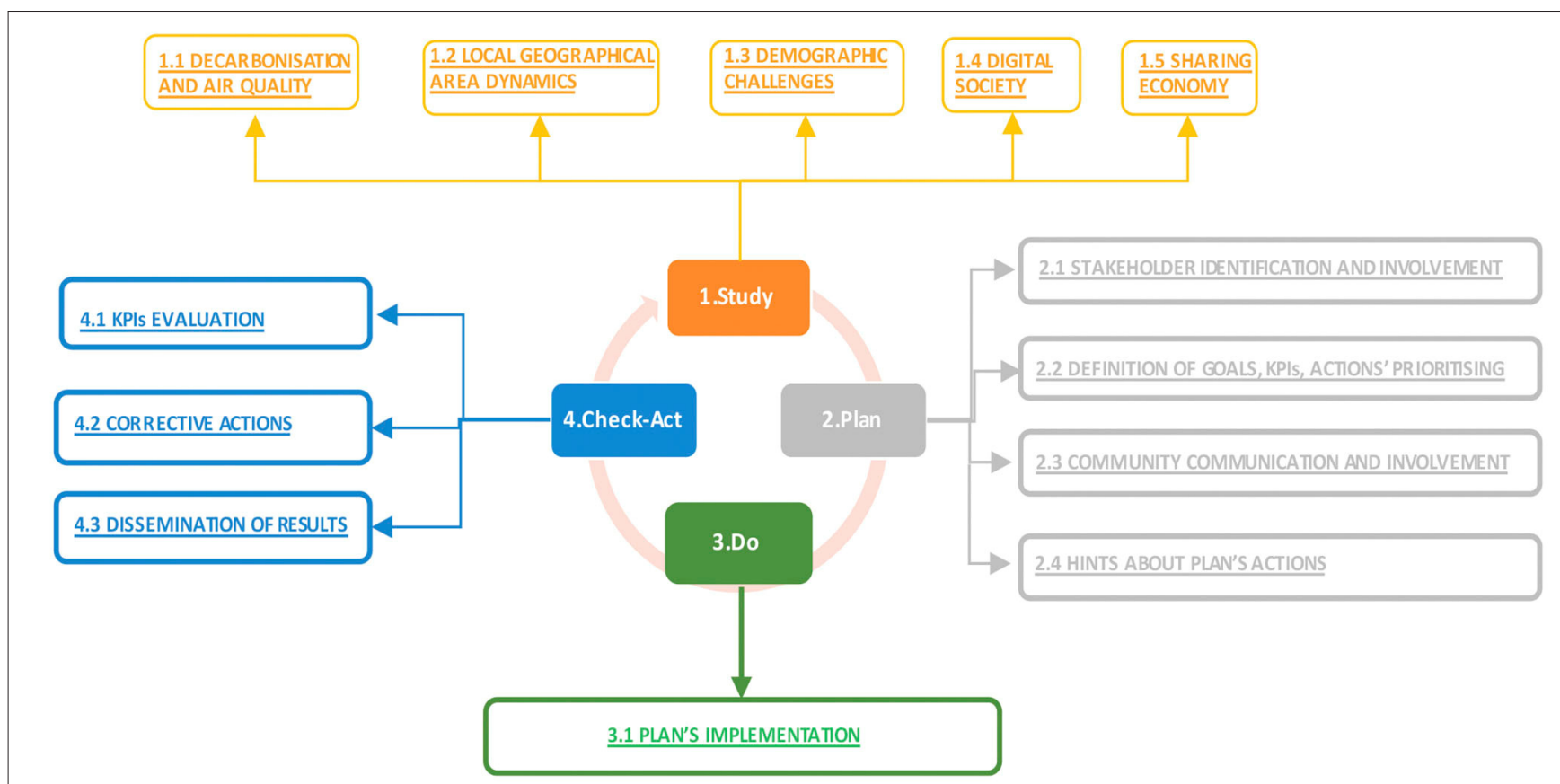

FIGURE 1 | Proposed CAMP-sUmp Action Plan overview (Regattieri et al., 2018).

serve as a guide for the design of the roadmap. There are different approaches to carry out this evaluation, and the use of questionnaires is a common one. This type of quantitative research is a rigorous scientific process designed to provide an accurate measure of public opinion (Grosvenor, 2000). The data collection is more structured, the sample size is larger and representative of the population, which allows for detailed and complicated statistical analysis (Lajunen and Ozkan, 2011), the research can generally be replicated or repeated, because it is reliable, and the analysis of the results is more independent. Qualitative survey methods are increasingly used in research and policy studies for the understanding of perceptions, attitudes, and behaviors of travelers, as a complement to more established quantitative surveys (Grosvenor, 2000). One of the advantages of qualitative data analysis techniques is that they lead to a rich and detailed contextual description of the phenomenon under investigation. However, analyzing qualitative information can be a difficult and arduous process (Clifton and Handy, 2001). By following a systematic process and paying attention to the issues of validity, consistency, and reliability in data collection and analysis, qualitative methods can have the same rigor and credibility as quantitative methods (Miles and Huberman, 1994).

SWOT analysis is a strategic planning tool used to identify and evaluate options that can lead to decision making. It is a twodimensional analysis that examines internal and external forces, both positive and negative (David, 1993; Helms and Nixon, 2010; Jonassen, 2012). It is considered a comprehensive method that studies the environment of the system and analyzes its interior. It is based on four components: strengths, related to the advantages of campus mobility tools, instruments and plans; weaknesses, related to disadvantages, insufficient capacities, lack of mobility policies, tools or instruments; opportunities, which refer to the overall impact on mobility and environment, staff and student satisfaction, quality of life, innovation and technology, human resource potential, urban and regional development and mobility policies, etc.; and threats, related to financial instruments, development costs, implementation and maintenance costs, legislation, complexity of communication between stakeholders, political implications, etc. This type of analysis has been used to evaluate participation in the design of sustainable mobility plans (Gil et al., 2011, 2015); to evaluate the use of the bicycle service (Ibeas et al., 2011; Bordagaray et al., 2015); and the use of electric vehicles (Raslavičius et al., 2015). A key procedure to analyze the current situation, needs, future challenges and priorities is to perform a gap analysis (GAP Analysis) to establish objectives, missions and goals (Wefering et al., 2014; Nolan and Anderson, 2015). Different researchers have implemented evaluation tools for this (Kose et al., 2014; Sdoukopoulos et al., 2016).

Based on the foregoing, a quantitative and qualitative study was carried out, as well as a SWOT and GAP analysis, a review of mobility measures in Mediterranean Universities, and of roadmap tools implemented in the context of the CAMPsUmp project; this allowed to know which roadmap should be developed. This paper presents the process for deciding what the roadmap should look like, its features, and the discussion of why it is transferable to the context of all Universities.

The universities have so far presented numerous actions to improve the mobility of their affiliates, but, however, there is no standardized/homogeneous method to help them select which of them are the most appropriate for their needs. So, 
this document aims to facilitate this process setting up a standard roadmap for developing mobility strategies appliable to University Campuses with different environmental, social and economic characteristics. It is worth to use this roadmap because it allows to obtain a strategic view of the whole process.

\section{MATERIALS AND METHODS}

The starting point for the development of the Roadmap was the exchange of good practices and knowledge on sustainable mobility between different universities, as well as the experiences of the CAMP-sUmp partners themselves.

First, an evaluation was conducted in order to identify the strengths and weaknesses of the current mobility planning practices implemented at the Universities, as well as the potential, the desired mobility objectives, and the characteristics of such mobility practices, following the guidelines of Wefering et al. (2014) for achieving a successful Urban Mobility Plan. For this purpose, the partners from the seven universities that constitute the CAMP-sUmp project (University of Catanzaro, National Technical University of Athens, University of Malta, University of Valencia, University of Split, University of Cyprus and University of Bologna) carried out a self-assessment procedure. First, a quantitative study was carried out to collect data on the mobility of users around the campus. In this regard, a questionnaire was developed, that included questions about current and intended mobility, mobility problems, proposed measures, mobility policies and tools. In order for the sample to be representative of all university users, participation was required from $10 \%$ of professors, $20 \%$ of administration staff, $20 \%$ of graduate students, $50 \%$ of undergraduate students. 1,090 questionnaires were collected through this procedure. The results of the quantitative study based on the questionnaires showed different strategies and measures depending on the location of each university. In universities located within urban areas, safety at intersections was the most critical measure, followed by measures to increase frequencies and to improve the density of the public transport network. For campuses located outside urban areas, the results indicated that measures focusing on public transport and road infrastructure should be taken in order to improve campus accessibility (Deliverable 3.2.1 CAMP-sUmp Project Papantoniou et al., 2017).

The next step was to conduct a qualitative analysis, whose aim was to collect qualitative information on the state of mobility within the campus, services and planning policies and tools on mobility flows, their integration within urban mobility and the situation of mobility to, from and within the campus. Seven universities provided structured feedback considering their university campus (see Table 1 ). The results indicated that, across all the examined campuses, students and employees are the most critical stakeholders, while the mobility situation of visitors has a secondary focus. Compared to decision-makers, local and regional authorities are usually involved in each campus mobility plan. A list of all campus mobility policies and tools at each university was analyzed. Car sharing and bicycle-related programs were the most commonly implemented practices.
Differences were observed considering the campus situation inside or outside the city (Deliverable 3.2.2 CAMP-sUmp Project, Papantoniou et al., 2017).

Third, a SWOT Analysis (strengths, weaknesses, opportunities and threats) was carried out based on a questionnaire administered to three to five experts for each university (university mobility and planning managers, technical representatives of local, regional and national public institutions, associate members and project administrators). The aim was to reveal the current state of campus mobility flows and the tools of sustainable mobility planning, as well as the implementation of SUMP in campus mobility planning. The strengths of campuses located in urban areas include easy accessibility through the use of public transportation system. In addition, students who live up to about $5 \mathrm{~km}$ from the campus have the possibility of going to the University in non-motorized modes of transport such as electric mopeds, electric scooters or bicycles. The strength of an out-of-town campus is that there is plenty of additional space for facilities and infrastructure, lot of parking spots, easy access by car and an improved quality of the environment for members. Opportunities and threats were common on campuses inside and outside of urban areas. Most campuses located near city centers face mobility problems due to traffic congestion, lack of parking space, use of active means of transportation, etc. Campuses outside urban areas face accessibility problems by public transportation. Mediterranean campuses are generally located near the city center or in suburban areas (Papantoniou et al., 2017).

A gap analysis was also carried out, by collecting qualitative data from mobility experts from the Campuses of the seven universities that constitute the consortium. Thirty-six interviews were conducted on the mobility gap to, from and within the Campus areas. The objective was to analyze the current situation of the campus in each thematic area, as well as to obtain a list of deficits and needs, future plans, challenges and priorities in these areas, as well as to provide a list of tools and policies for sustainable mobility. The covered areas were parking management, smooth mode infrastructure, public transport, motorized private transport, road infrastructure, environment and energy, mobility management, transport infrastructure and merchandise management, information and tools of communication technologies and mobility plans. Mobility experts were asked about the current situation, existing measures, tools and policies, as well as problems, needs, plans and future priorities. The results indicated differences among Universities depending on whether they were located inside or outside the city. Location within the city is an advantage for the implementation and planning of strategies for public transport, road infrastructure and ICT tools.

The analysis identified a significant gap in terms of environmental impact and energy issues. The challenge in this case is the implementation of strategies to protect the environment, clean vehicles, charging stations for electric vehicles, and the use of small vehicles for indoor mobility. The absence of public transport for mobility within the campus is common, since the buildings are close to each other. Strategies aim at improving pedestrian safety measures at crossings by 
TABLE 1 | Participants.

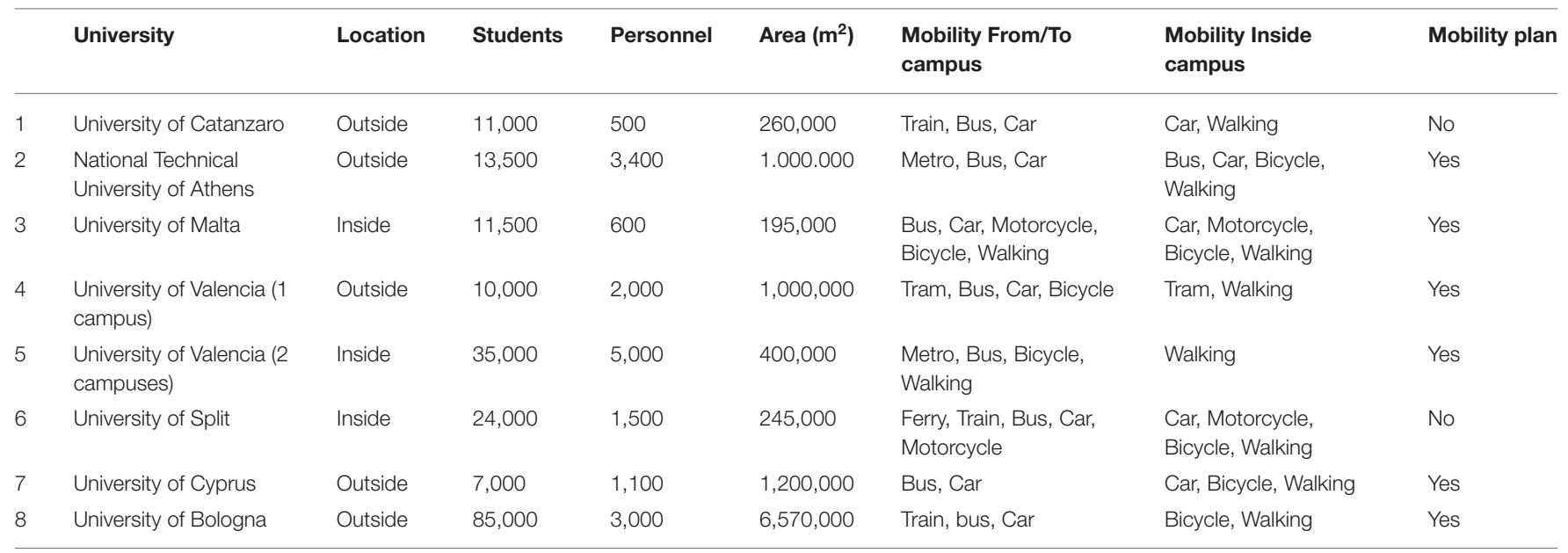

Seven Universities were examined. (Extracted from Deliverable 3.2.2 CAMP-sUmp Project, Papantoniou et al., 2017).

establishing bicycle rental services. Parking management and the role of ICT tools to support campus sustainable mobility plans were the most relevant areas. Campuses located outside the city or in suburbs had the best infrastructure in terms of road transport. These are new areas, with high quality road infrastructure.

The gap analysis found that the gap is in public transport and mobility management. There is a need to implement various measures and policies to improve density, expansion, comfort (stops, stations and vehicles) and security (surveillance) of the public transport network, as well as ICT to improve information for passengers, and the ticketing systems, in order to implement intelligent transport systems and increase frequencies. Also, lighting, pavement, adaptation of access for disabled users, and signaling can be improved. To reduce the gap in mobility management, some proposals include the inclusion of information and advice on travel options, ICT tools, a mobility center, and the raise of awareness in order to promote and support sustainable mobility. Car-sharing and carpooling systems were missing, and they were important ones (Deliverable D3.3.2; Papantoniou et al., 2017, 2018).

A comprehensive review of sustainable mobility measures and actions existing in different Mediterranean universities was carried out in order to verify the differences and similarities between them, while different SUMPs were proposed. The CIVITAS ${ }^{3}$, EPOMM, CRUE, CRUI, UMOB LIFE ${ }^{4}$, SMILE PROJECT, STARS projects were reviewed, and information was obtained from the conference of chancellors from the involved universities, as well as from congresses on sustainable mobility in Universities. (Deliverable 3.3.3, Papantoniou et al., 2017; Deliverable 3.5.1, Tormo Lancero et al., 2018). Some examples of universities that have implemented mobility plans based on the city's SUMPs are: The Polytechnic University of Turin in Italy (Vlahogianni et al., 2018) and the University of Barcelona

\footnotetext{
${ }^{3}$ http://civitas.eu/mobility-management/planning;

http://civitas.eu/tool-inventory

${ }^{4}$ http://2017bcn.u-mob.eu/es/
}

(Miralles-Guasch and Domene, 2010). The University of Malta has had a Green Travel Plan (GTP) in place since 2010 (Attard et al., 2011). The University of Glasgow has been operating a Green Transport Policy since 2006, with objectives that are easily identifiable and in line with the SUMP approach. The University of Bristol (United Kingdom) (University of Bristol, 2017), the University of La Coruña (Deliverable 3.3.3, Papantoniou et al., 2017), the University of Rome, The National Technical University of Athens (Papantoniou et al., 2018) and the University of Milan (Silva and Ferreira, 2008) have all been carrying out mobility plans as well.

Some authors propose mobility solutions to help university mobility managers, in terms of shared electric vehicles and infrastructures (Longo et al., 2014), and they analyze the impact of some sustainable solutions on campuses and their integration with the city (Kennedy et al., 2005; Lukman and Glavič, 2007; Nejati and Nejati, 2013; Pitsiava-Latinopoulou et al., 2013; Al-Mosaind, 2014). Several studies have shown that on university campuses there are daily commuters who travel longer distances, and for whom the use of private cars prevails over non-motorized means of transport (MirallesGuasch and Domene, 2010). The location of the campus, in the city center, on the outskirts or outside the urban area, is a key element when establishing mobility measures. Other studies corroborate that on campuses located outside the city mobility depends largely on individual transport, due to the inefficiency of the public transport system and other alternative modes (Fiadeiro, 2008). Therefore, it is important to propose innovative approaches and move toward appropriate forms of sustainable mobility (Silva and Ferreira, 2008). Finally, the study on the European urban transport roadmap 2030 (De Stasio et al., 2016) was consulted.

\section{RESULTS}

The results of the review of good mobility practices in Universities, the quantitative and qualitative study, as well as the SWOT and the GAP analysis, indicate that the context of 
universities can be different, depending on the characteristics of the city, the characteristics of the University (number of students, number of employees, resources, actors involved), the administration, the transport networks and the infrastructures: therefore, the objectives and measures must also be adjusted to each University. The main differences were found in the location of the University. More specifically, the type of measures, policies and implemented planning tools were different depending on the location of the University (within or outside the urban area). The strengths and weaknesses also varied among universities in terms of location; however, the opportunities and threats were similar. In addition to the location, the review of the measures currently implemented in Universities showed that, although some measures were common, some of them were different depending on the context and characteristics of the University itself (Tormo Lancero et al., 2018). This variability implies that, although the similarities between campuses are more numerous than their differences (and, therefore, mobility problems must be approached in a similar way), in order for the Roadmap to be useful, each University should consider its particular needs and conditions. Each University also needs to establish its priorities, its own personalized objectives, as well as to choose the measures, the time and resources to achieve them.

Based on what we abovementioned, a standard roadmap for a successful implementation of such an action plan in university campuses with different scenarios and characteristics is proposed in this study, considering different environmental aspects, socio-economic conditions and environments, to ensure transferability. The result will become a standardized and customizable tool that contains methodology and common elements among all Universities, so that each University can adapt it to their circumstances. As part of the development of the standard roadmap, a series of questions are considered that planners must answer based on their self-assessment. This roadmap takes into account the steps of the action plan, sets out the objectives to be achieved, in the short, medium and long term, the required tools and measures to be implemented, the actors, the impact, the budget, costs and threats for the implementation of the measures, the development time, the degree of achievement, and, finally, it guarantees the commitment of those responsible of implementing such practices, as well as of decision makers, for the Implementation of the Action Plan. This tool contemplates the strategic lines of sustainable mobility along which universities should act: transversal aspects; walking; cycling and other smooth and clean modes; public and intermodal transport; private transport and parking. For each strategic line, it is possible to include objectives and measures from different areas of intervention, such as social measures and mobility management; infrastructure measures; measurements on the vehicle; ICT tools (Tormo Lancero et al., 2018).

CAMP-sUmp Roadmap is made up of two complementary documents. The first (tactical document) allows for a global vision of the plan in a template, and the second (operational document) one allows for the specification of the work detail for each strategic line of mobility, in an interval of given time.

\section{Tactical Roadmap}

It is supported by instructions on how to fill in the information fields, information on how to identify the current situation of each university, and a catalog of the most promising actions for sustainable mobility in Universities, obtained from the results of the CAMP-sUmp project and from the review of mobility measures, actions and plans in Universities (Tormo Lancero et al., 2018). This catalog provides a list of examples of goals, objectives, transport, environmental, economic, personal and safety indicators, types of social measures, mobility management, infrastructure, vehicle and ICT measures implemented by the Universities for each strategic line (cross cutting, walking, cycling or clean modes, public transport, private transport and parking), as well as the impact, the costs of each measure and the possible threats and difficulties. The catalog also includes a section with the most common measures implemented among Universities, and an example of a completed roadmap. This catalog is a framework of available tools that each University can consult to create a realistic roadmap with measures adjusted to its objectives, resources, budget and desired impact. The CAMPsUmp project has also provided a review of the most important measures of information and communication technologies, thus obtaining a comprehensive ICT model that can help Universities to complete their roadmap in the sections related to this question (Campos et al., 2018, 2019).

The tactical part of the roadmap allows for the introduction of the diagnosis of the starting context, as well as the objectives and milestones that are intended to be developed throughout the entire Plan, differentiating between the short, medium and long term, and the general actions for each strategic line from the different areas of intervention. It also allows universities to indicate an estimation of the impact and the cost of the proposed measures. The information can be introduced in an electronic template available at this link (https://CAMP-sUmp.interregmed.eu/what-we-achieve/roadmap/).

The template of the tactical or global document of the Roadmap consists of the following sections (see Figure 2):

- Name. In this section, the name of the University that is going to complete the Roadmap must be indicated.

- Key. The colors indicate the scope of intervention for every type of measure to be established for each strategic line of mobility. The measures can be social management and mobility, infrastructure, vehicle and ICT. For each measure, the following information are included: - box I (Impact) and box $\mathrm{C}$ (Cost), in which the person will indicate whether the expected impact and cost of the measure is Low (L), Medium $(\mathrm{M})$ or $\operatorname{High}(\mathrm{H})$.

- Initial conditions or context: The first step to plan a roadmap begins with a descriptive analysis of the initial conditions from which the University starts. This analysis will mark the short, medium and long-term objectives that define the horizon of the plan, as well as its strategic lines. Each university must identify the mobility problems it faces, and define the objectives it would like to achieve, taking into account the specifications of the Action Plan. The elements to consider in this section are: the type of university; the population; the 
Tormo-Lancero et al.

Sustainable Mobility Roadmap for Universities

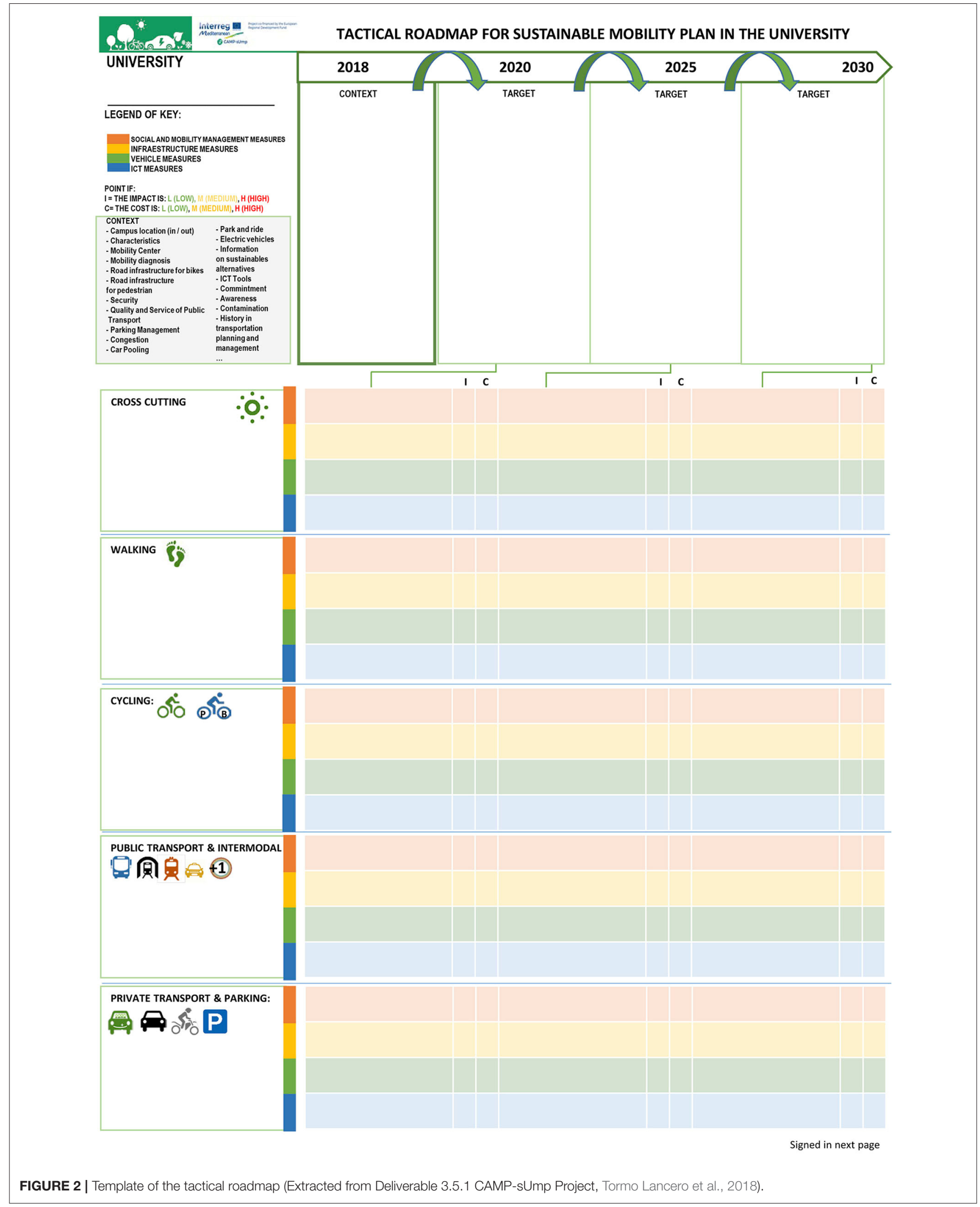

Frontiers in Sustainable Cities | www.frontiersin.org

7

January 2022 | Volume 3 | Article 668185 
economy-income; traffic congestion; air quality and pollution; the public transport service; the use of the private vehicle; infrastructure and safety for pedestrians and cyclists; the lanes reserved for bicycles and public transport.; the use of car sharing; parking and using the metro; electric vehicles; parking fees; parking network and standards; information on sustainable mobility alternatives; ICT tools; History of Transportation Planning and Management; decision-makers commitment; awareness; facilities to improve sustainability, and motorization rate. To carry out the self-assessment of the initial conditions and determine the main objectives, different approaches can be used: methodology of surveys to be filled out by users of the University, interviews with experts, SWOT and GAP analysis, review of roadmaps defined by other Universities and previous knowledge. The catalog of Deliverable 3.5.1 of the CAMP-sUmp project (Tormo Lancero et al., 2018) can be greatly helpful in this regard. Another useful tool that could be used for this purpose is the European Commission's "Urban Transport RoadMap", which provides a perspective to define the objectives that must be prioritized.

- Goals / Milestones: In this section, after obtaining the diagnosis of the initial and desired situation, each University will present the objectives or milestones that it intends to achieve in the short, medium and long term.

- Selection of measures applicable in the short, medium and long term: This section contains a matrix to specify the actions or measures that the university wishes to carry out in the short, medium and long term. There are a number of threats to consider when applying these measures that are specified in the catalog. The strategic lines cover the modes of transport to which the measures will be applied, and they are the pillars of sustainable mobility: cross cutting; walking; cycling; public and intermodal transport; private transport and parking. The measures for each strategic line or mode of transport to which it is directed can be classified into measures of social management and mobility; measures on infrastructure; measures on vehicles; and ICT measures. Each one of the actions is identified by colors. It should be taken into account that the same measure can affect different modes of transport, and two approaches could even overlap. One aspect that has been taken into account as a cross-cutting issue from the perspective of gender equality is to improve the safety conditions of girls and women while they travel to, from and within the campuses, in order to promote and increase their active mobility by reducing gender-specific risks. The work approaches or policies implemented to develop these strategic lines are the following:

- Social approach and mobility management. From this perspective, the intention is to change the behavior of users through information and promotion actions. It includes Mobility Management, regulation and incentives. This approach uses a series of measures designed to provoke a more sustainable behavior in the mobility of the university members. Social measures include information programs, awareness, promotion of sustainable trips and dissemination. They have an effect on all modes of transport, reducing congestion, improving safety and having a positive impact on air quality. Their cost is low, and many measures are relatively quick to implement, both in the short and medium term. Mobility Management refers to political, bureaucratic, economic and social procedures, creation of figures, commissions, committees, parking management through the use of incentives, road fees, shared cars...

- Infrastructure. This aspect includes investments in the planning and construction of infrastructure and means of transportation, as well as land use planning. It involves changing the urban environment. These strategies should improve accessibility and reduce congestion and emissions. Infrastructure measures require a significant financial effort and take time to implement, which is why they are generally included as long-term strategies.

- Vehicle: In this category, we group the measures that refer to investments in technology and specific characteristics of the vehicle;

- ICT. This section defines the information and communication technology measures to be applied;

- Impact and costs: In cell "I", L, M or H there will be indications on whether the expected impact of the measure is low, medium or high. In cell " $C$ ", the university will indicate whether the expected cost of the measure is low, medium or high. A decision must be made about which interventions are to be implemented, as there can be great a variability in terms of investment and impact.

Social aspects and mobility management measures tend to have lower economic costs, with rolling stock and infrastructure measures being the ones that usually involve the most investment, in this order. Plans aimed at promoting sustainable modes of transport and at regulating the use of private vehicles, as well as the transport of goods, have a lower cost than those aimed at modifying infrastructure. However, the policy and social costs of the early and intermediate phase can be very high, so the information, awareness and participation of users in the plan is a fundamental aspect. For example, parking regulation and pricing are very sensitive measures, if we consider the political side, as parking is often free and perceived as a right. Therefore, any attempted modification may result in strong opposition. The European Commission study, 2016 Ref. MOVE / C1 / 2013-1882 provides an approach to these policies, as well as on the impact they have on the modes and the costs of implementation. This analysis provides guidance and information on the costs and benefits of different strategies, which in turn serves as a support tool in making decisions about the policies to be implemented. This information can be useful for Universities when deciding the type of measures to use.

- On the back of the document, the signature of the decisionmakers must be stamped, indicating the commitment to the development of the Roadmap.

\section{Operational Roadmap}

The tactical document is accompanied by the operational roadmap (see Figure 3).

In the operational document, the information is presented in five independent templates, one for each strategic line of 


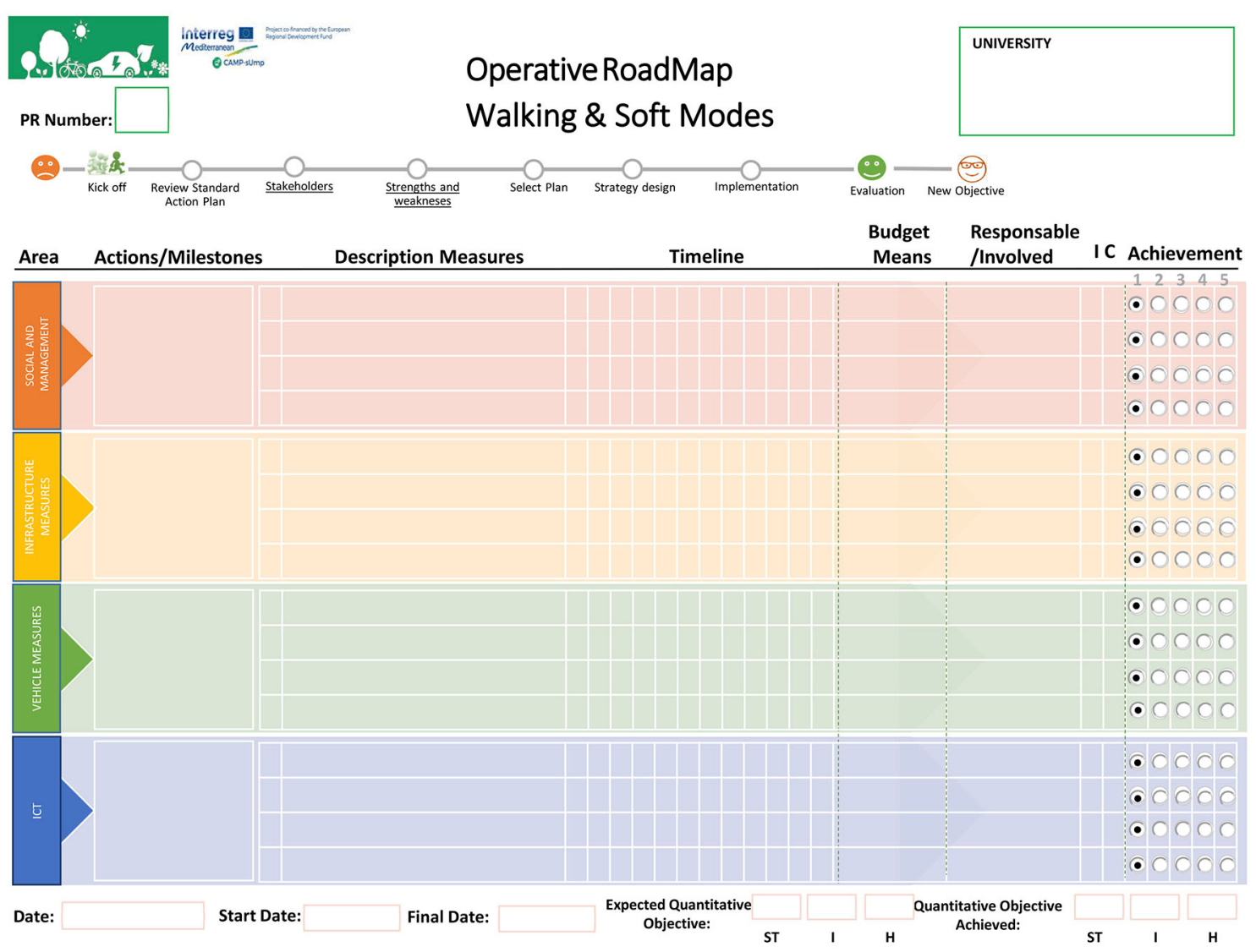

FIGURE 3 | Template of an operational roadmap for the strategic line of walking (Extracted from Deliverable 3.5.1 CAMP-sUmp Project, Tormo Lancero et al., 2018).

sustainable mobility: cross cutting; walking and soft modes; cycling; public transport; private transport and other smooth and clean modes. This document expands and gives more detail on the objectives and actions to be followed for each strategic line during a given period. It includes a reminder of the steps needed to prepare the plan, and it organizes the measures and actions for each strategic area according to its type. It includes information about the University; Period of realization; Area or line of action (social and mobility management, infrastructure, vehicle, ICT); Description of each Measure with its Implementation and the Realization Time Schedule of its implementation, Budget and Means, People responsible for and involved in this measure, Impact and Cost, Monitoring of Achievements or degree of achievement of each action (from 1 to 5); Start date and end date of the period for carrying out these actions; expected target (from 0 to 100) in the short, medium and long term; objective achieved (from 0 to 100) in the short, medium and long term; Formal commitments: it is important that managers and collaborators sign the roadmap as a sign of commitment.

This roadmap must be accompanied by a communication plan whose objective is to inform about the implementation of the action plan and achieve a positive attitude toward the mobility changes, prioritizing sustainable mobility. To develop the communication plan, it is necessary to draw some main objectives, a strategy, and to have people who are willing to participate. The communication plan should be designed in several phases. Some important aspects to consider are ICT, social media, social networks, alerts and messages. Finally, it is important to follow up to evaluate its impact.

\section{DISCUSSION}

In this paper, an extensive review on mobility measures in Universities was conducted, while an important gap emerged regarding the lack of homogeneity in the procedure, tools and strategies used to manage and improve mobility in Universities. In particular, it turned out that this gap is due to the lack of an action plan and a standardized route for the improvement of sustainable mobility in Universities. This is one of the relevant findings of this work. Considering this gap, this paper is aiming at creating a standardized roadmap, that universities can use, share and utilize to learn from each other, thus helping the implementation of a successful action plan. This is undoubtedly the main finding. The goal is to achieve a more sustainable, competitive and safe mobility system in universities, allowing for a greater accessibility and a better shared space through smooth modes. The strategic lines are mainly oriented toward less car use and more use of public transport, cycling, and walking. This will 
have a positive impact on the quality of life of students and staff, as well as on the environment.

Initially, we had to decide whether it was convenient to develop a standardized Roadmap for all Universities, or whether, on the other hand, it was preferable to consider their particularities instead. We started from the hypothesis that there is wide variability in the contexts of Universities, and that a common Roadmap may not be useful for all contexts. One of the main findings of this work was that. although there are lots of similar problems as well as implemented mobility measures in Universities, they all start from a specific context; therefore, adjusting the objectives and priorities of each university regarding mobility is required. These results oppose the development of a standardized roadmap that is valid for all Universities.

This conclusion was reached from a review of mobility strategies implemented by Universities, and from a selfevaluation of the Universities participating in the CAMP-sUmp project through a quantitative and qualitative study, a SWOT Analysis and a GAP analysis. It was observed that a differentiating aspect between Universities was the location of the campus, within or outside the urban area. Its location determined different types of interventions. This led us to discuss the need of establishing a different roadmap depending on the location of the Universities. The most remarkable result was that, in campuses located within the urban area, the percentage of users who traveled on foot and by bicycle was higher than in campuses located outside the city. Therefore, in this case pedestrian safety at intersections is the most critical need, followed by public transport measures in terms of increased frequencies, density and extension of the public transport network. Parking management is also a crucial issue to consider in these cases. In summary, the most important actions that must be taken are: safety at crossings, increasing measures in public transport-in terms of increasing the frequencies, density and extension of the public transport network -, and parking management, due to parking problems. Other necessary actions in these campuses are: improving the road infrastructure (due to the lack of a safe access by bicycle and on foot), mobility management, and the use of ICT tools.

According to the SWOT analysis, the strength of these campuses, in comparison with those located outside the city, is the easy accessibility through the city's Public Transportation System. The costs of public transport services are lower than in Universities located in the outskirts. Users who live within up to five kilometers from the university the opportunity to access the facilities on electric mopeds, electric scooters, bicycles, or on foot. There is a greater awareness among the university population on the need for a SUMP, and the staff and students live relatively close. The fundamental problems of campuses located within the city are: traffic congestion, narrow streets, lack of parking space, lack of use of active transport modes, the perimeter of the campus is not very accessible in smooth ways and it is difficult or impossible to modify arterial lines. The location of the university buildings is more dispersed and there are limitations in space. In campuses located outside urban areas, the results indicate that the main measures should focus on public transport and road infrastructure, in order to have a better accessibility to the campus by bicycle and on foot. Other necessary actions are mobility management and parking management. On these campuses, cars are the most preferable mode of transportation regardless of the length of the trip. The biggest problem is the duration of the trip on public transport, which can be up to four times longer than by private transport. Thus, the fundamental measures in these campuses must be directed toward public transport and adequate infrastructure for the use of smooth access roads to the campus. The students' residence is usually at a longer distance from the University and there are numerous students who come from different parts of the territory every day. The main strength of these campuses compared to the previous ones, according to the SWOT analysis, is the existence of many free and unrestricted parking spaces, which can otherwise represent a threat to sustainable mobility. The fundamental problem is accessibility, due to the lack of competitive modes of transport that are an alternative to private vehicles. Other strengths that stand out in this type of Campuses, in comparison with a Campus within the city, is that they are usually located in developed areas, with significant flows of people interested in going there, with a wide and accessible space, plenty of space to go on foot and by bicycle, multiple accesses by car, pedestrian networks and bicycles lanes to circulate within the campus, and a high availability of parking spaces. They are relatively new campuses with a high-quality road infrastructure.

Regarding the weaknesses presented by these campuses, there is a majority use of private vehicles with low occupancy. There is also a demand for public transport, but the low level of service and the lack of good intermodality leads to low utilization, which in turn produces a reduction in the provision of public transport service due to low demand. In addition, the distance from the public transport stops to the University is usually long, occurring mainly for budgetary reasons. Therefore, it is necessary to respond to transport demands according to the needs of students and staff, in order to improve the existing mobility services. For this purpose, it is necessary to increase investments in sustainable transport connections between the urban area and the campus. On the other hand, in these campuses there is a low demand for transport on foot and by bicycle, and traffic is usually high to access the University. Also, the infrastructure is inadequate for walking or biking. Therefore, measures should be aimed at improving the quality of the public transport service, infrastructure, and vehicles, as well as the awareness of transport options alternative to cars, which include public transport, carpooling, bikes, and going on foot. Mobility management is also something that can be improved using ICT tools. However, ICT tools for mobility are more expensive due to the large distances they have to cover.

It was also observed that the strength of universities is knowledge, and their most prominent weaknesses are the lack of funds to invest in soft modes, the lack of planning, programming and communication policies, the lack of coordination between university and local authorities, the difficulties of coordinating so many people, the lack of information and awareness about sustainable mobility, the consideration of owning a private vehicle as a symbol of social status, the limitations in the 
governance of public transport, the lack of management of the campus, and the ignorance about the use of soft modes for the journey. In terms of opportunities, reducing car use and traffic congestion are the main aspects that should be considered. The threats to all this is the slowness of bureaucracy that lengthens both the planning and execution time. On the other hand, the size of the University is an important aspect to take into account in the implementation of a SUMP. Therefore, a small center may be easier to manage, but nevertheless, in this case, the capacity of the University may be insufficient due to its size, or to the lack of specialized personnel. Similarly, if a University has only one campus, planning is much easier. The social, economic, and environmental context, the starting budgets, financing, etc. are fundamental for the establishment of priorities, objectives and type of measures to be implemented. This situation confirmed our hypothesis that, although the similarities shared by the campuses are numerous, it is necessary to take into account their differences in order to obtain a valid Roadmap.

\section{CONCLUSIONS}

The review has shown that although the universities performed numerous activities to boost sustainable mobility, they, however, did not have an standardized methodology to develop plans with such goal in a comprehensive way. Furthermore, the activites were focused on the in-out mobility, i.e. commuting, but they did not contemplated mobility within the campus (Tormo Lancero et al., 2018; Papantoniou et al., 2020). Aditionally, a qualitative-quantitative study, SWOT, GAP, and a review of the mobility measures in the Mediterranean universities and of the road map tools. The results showed that each university has certain characteristics that require different mobility solutions, as suggested by Gory et al. (2012) the campus localization is a key element to consider. However, it is still possible to establish a set of guidelines and steps to follow and common aspects for all the universities that might facilitate the implementation of the mobility plan. No scientific contribution providing an standardized road map was found that developed sustainable mobility plans for the university mobility managers.

The first contribution of this work is the introduction of an standardized road map permitting the successful implementation of sustainable mobility plans in the universities. This plan is customizable and can be transferred easily.

This standard Roadmap may be helpful for decision makers, which includes general guidelines for all Universities, elastic and customizable, so that each University can adapt it according to its needs, circumstances, objectives, measures, stakeholders, calendar and budget. This advances the current views, since until now there was no specific document available for Universities. The Roadmap is designed as a fillable template that includes the steps to follow, and a series of elements that Universities can complete and customize based on their particularities from a self-assessment of the starting point. Thus, the road map begins with an assessment of the issue, following the guidelines by Wefering et al. (2014) Canitez (2020), Foltnová et al. (2020). The methodology that we propose is the realization of a quantitative and qualitative study, a SWOT analysis and a GAP analysis. The road map guarantees the cooperation, the dialogue, the capability, and the commitment of the decision makes on the planification of sustainable mobility.

The roadmap consists of two documents: a tactical document that offers a global and sequential vision of the plan, and an operational document, which details the measures to be established for each time span. The fundamental strategic lines that the Roadmap deals with are: cross cutting, walking, cycling, public and intermodal transport, private transport and parking management. The policies or work approaches are: social and mobility management measures, infrastructure measures, vehicle measures, and ICT measures. Some aspects that are as important as the specific objectives are: the type of measure, the cost and impact, the desired and achieved goals, the implementation dates and the signing of the commitment by the decision-makers and by those involved in each action. This road map has mobility solutions from and to the campus. Due to its flexibility, we believe that it guarantees the transferability to university campus with different environmental and socioeconomic characteristics.

Another novelty of this work is that from the review and selfevaluation process of the mobility of Universities in the context of the CAMP-sUMp project, a compilation of good practices and experiences of mobility has been created. This catalog presents objectives, measures, cost and impact indicators, limitations and threats, and a summary of the main, most shared measures implemented by Universities. This makes it a good source of consultation on mobility strategies for Universities, and it can be a good support instrument to complete the university's Roadmap, as well as to establish objectives and measures in the short, medium and long term. (Deliverable 3.5.1 of the CAMP-sUmp project, Tormo Lancero et al., 2018).

The methodology to design the Roadmap through a quantitative, qualitative study, GAP and SWOT analysis is a clear contribution to academics. The review of good practices in Universities is relevant as a source of consultation to propose a personalized roadmap.

The limitations of this research are that it has been developed in the Mediterranean area. Regarding its suitability for Emerging Countries, although it has been developed based on a review and analysis of Universities in the Mediterranean area, we sense that the instrument should be useful: in fact, the strategic lines of mobility, and the policies to deal with them, are universal. Also, the roadmap is customizable, and it allows universities to set priorities and to decide the type of measures they wish to implement, based on their own self-evaluation. The catalog that accompanies the Roadmap could be improved or expanded based on good practices in these countries. This is a necessity, and we launch the challenge that it should be done.

Finally, we postulate the theory that the use of this tool in Universities can promote the exchange of good practices among them, and it can lead to the development of innovative mobility 
solutions that can be transferred to broader contexts, such as cities and regions.

\section{DATA AVAILABILITY STATEMENT}

The data indicated in the manuscript were obtained in the context of the CAMP-sUmp project (CAMPus sustainable University mobility plans in MED areas) Project Nb. 1MED 15_2.3_ M1_238. Project Interreg Med co-financed by the European Regional Development Fund. The project link is: https://camp-sump.interreg-med.eu/. The data was obtained from surveys and interviews in the context of this project.

\section{AUTHOR CONTRIBUTIONS}

All the authors have participated in the development of the work, the writing of the manuscript, revision, and translation of the manuscript.

\section{REFERENCES}

Al-Mosaind, M. (2014).Traffic conditions in emerging University Campuses: King Saud University, Riyadh, Saudi Arabia. J. Sustain. Dev. 7, 204. doi: 10.5539/jsd.v7n6p204

Attard, M., Bajada, T., Gauci, P., Camilleri, L., and Spiteri, C. (2011). Travelling Smart - Green Transport Plan for The Msida Campus, University of Malta. Msida: University of Malta Institute for Climate Change and Sustainable Development.

Balsas, C. (2003). Sustainable transportation planning on college campuses. Transport. Policy. 10, 35-49 doi: 10.1016/S0967-070X(02)00028-8

Bordagaray, M., dell'Olio, L., Ibeas, A., Barreda, R., and Alonso, B. (2015). Modeling the Service Quality of Public Bicycle Schemes Considering User Heterogeneity. Int. J. Sust. Transpor. 9, 580-591. doi: 10.1080/15568318.2013.838722

Campos, E. D., Tormo Lancero, M. T., Valero Mora, P., Papantoniou, P., Vlahogianni, E., Yannis, G., et al. (2018). Deliverable D3.5.2: ICT tools, models and requirements for communication between different actors and planning instruments "CAMPus sustainable University mobility plans in MED areas".

Campos, E. D., Tormo Lancero, M. T., Valero Mora, P., Papantoniou, P., Vlahogianni, E., and Yannis, G. (2019). Shaping the monility in university campuses throughout ICT solutions. International Scientific Conference on Mobility and Transport "Transportation Systems of the Future" Munich, 11-12 September 2019.

Canitez, F. (2020). Transferring sustainable urban mobility policies: An institutional perspective. Transport Policy, 90, 1-12. doi: 10.1016/j.tranpol.2020.02.005

CIVITAS. (2020). Available online at: http://civitas.eu/

Clifton, K., and Handy, S. (2001). Qualitative Methods In Travel Behaviour Research. Conference on Transport Survey Quality and Innovation. South Africa: Kruger National Park.

CRUE, 2013. Datos obtenidos a partir de la encuesta universitaria sobre buenas prácticas en movilidad sostenible y accesibilidad. Grupo de Trabajo Universidad y Movilidad. Comisión Sectorial de Calidad Ambiental, Desarrollo Sostenible y Prevención (C.A.D.E.P.).

CRUI. (2015). (The Conference of Italian University Rectors). Available online at: http://www.crui.it/rus-rete-delle-universita-per-la-sostenibilita.html

David, F. (1993). Strategic Management, 4th Ed. New York, NY: Macmillan Publishing Company.

De Stasio, C., Fiorello, D., Fermi, F., Hitchcock, G., and Kollamthodi, S. (2016). Study On European urban transport roadmaps 2030. Urban transport policy roadmaps. European Platform on Sustainable Urban Mobility Plans. Urban Transport ROADMAPS: Available online at: http://www.eltis.org/mobilityplans/project-partners/european-urban-transport-roadmaps-2030

\section{ACKNOWLEDGMENTS}

Thank to the research project CAMP-sUmp (CAMPus sustainable University mobility plans in MED areas) Project Nb. 1MED 15_2.3_M1_238, and to the project partners, without whom this work would not have been possible. CAMP-sUmp is a European research project co-financed by the European Regional Development Fund, aimed at improving planning instruments for sustainable urban mobility through innovative strategies that manage the flow of mobility of university campuses and their integration with urban areas. Available at: https://CAMP-sUmp.interreg-med.eu/. Thanks to the Ministry of Science and Innovation, Ministry of Universities in the State Plan for Scientific and Technical Research and Innovation 2013-2016 (Government of Spain), and thanks to the co-financing of María Teresa Tormo Lancero as Technical Personnel to Support R + D + I (PTA-201510596-I).

EPOMM. (2012). Available online at: http://www.epomm.eu/index.php ETSC. (2020). The Impact of Covid-19 Lockdowns on Road Deaths in April 2020 (PIN Briefing). Available online at: www.etsc.eu/PINCovid19

European Union. (2011). White Paper on Transport. Available online at: https://ec europa.eu/transport/sites/transport/files/themes/strategies/doc/2011_white_ paper/white-paper-illustrated-brochure_en.pdf

Fiadeiro, P. (2008): A mobilidade sustentovel aplicada aos equipamentos escolares - o caso do Polo II da Universidade de Coimbra, Dissertatto de Mestrado em Engenharia Civil, especialidade de Urbanismo, Transportes e Vias de Comunicatto, FCTUC, Departamento de Engenharia Civil, Universidade de Coimbra, Coimbra.

Foltnová, H. B., Vejchodská, E., Rybová, K., and Kveton, V. (2020). Sustainable urban mobility: One definition, different stakeholders' opinions. Transpor. Res. Part D: Transport Environ. 87, 102465. doi: 10.1016/j.trd.2020. 102465

Gamberi, M., Bortolini, M., Pilati, F., Regattieri, A. (2015). Multi-Objective Optimizer for Multi-modal Distribution Networks: Carbon Footprint and Delivery Time. Using Decision Sup-port Systems for Transportation Planning Efficiency. 330 doi: 10.4018/978-1-4666-8648-9.ch013

Gil, A., Calado, H., and Bentz, J. (2011). Public participation in municipal transport planning processes-the case of the sustainable mobility plan of Ponta Delgada, Azores, Portugal. J Transport Geograp. 19, 1309-1319. doi: $10.1016 /$ j.jtrangeo.2011.06.010

Gil, A., Fonseca, C., Calado, H. (2015). Participatory planning for the definition of sustainable mobility strategies in small islands. Sustainable Transportation in Natural and Protected Areas. Oxford, UK: Routlege. p. 181-192, 304.

Gori, S., Nigro, M., and Petrelli, M. (2012). The impact of land use characteristics for sustainable mobility: the case study of Rome. Eur. Transp. Res. Rev. 4, 153-166. doi: 10.1007/s12544-012-0077-6

Grosvenor, T. (2000). "Qualitative research in the transport sector. Resource paper for the workshop on qualitative/quantitative methods," in Proceedings of an International Conference on Transport Survey Quality and Innovation. Paper Presented at the Grainau, Germany, Transportation Research E-Circular, Number E-C008. p. 1-20.

Haas, M., de, Faber, R., and Hamersma, M. (2020). How COVID-19 and the Dutch "intelligent lockdown" change activities, work and travel behaviour: Evidence from longitudinal data in the Netherlands. Transpor. Res. Interdisciplin. Perspect. 6, 100150. doi: 10.1016/j.trip.2020.100150

Helms, M., Nixon, J. (2010). Exploring SWOT analysis-where are we now? A review of academic research from the last decade. J. Strategy Manage. 3, 215-251. doi: 10.1108/17554251011064837

Ibeas, A., dell'Olio, L., and Montequín, R. B. (2011). Citizen involvement in promoting sustainable mobility. J. Transport Geography. 19, 475-487. doi: 10.1016/j.jtrangeo.2010.01.005 
Jonassen, D. (2012). Designing for decision making. Educat. Tech. Res. Dev. 60, 341-359. doi: 10.1007/s11423-011-9230-5

Kennedy, C., Miller, E., Shalaby, A., Maclean, H., and Coleman, J. (2005). The four pillars of sustainable urban transportation. Transp. Rev. 25, 393-414. doi: 10.1080/01441640500115835

Khashaypoor, M., Da Silva, F. N., and Mamdoohi, A. R. (2021). An Overview of Challenges and Plans to Improve Urban Mobility: A Case of Approach and Strategies in Tehran. Int. J. Transp. Engg. doi: 10.22119/ijte.2021.238314.1524

Kose, P., Gal-Tzur, A., Sheety, E., Mezghani, M., Sdoukopoulos, L., Boile, M., Mitropoulos, L., Lah, O. (2014). Assessment of city needs and gap analysis, SOLUTIONS- Sharing Opportunities for Low Carbon Urban Transportation.

Lajunen, T., Ozkan, T. (2011), B. Porter's Handbook of traffic psychology, Chapter 4.

Longo, M., Roscia, M., Lazaroiu, G.:, Innovating multiagent systems applied to smart city. Res,. J., Appl. Sci. Eng. Technol. 7, 4296-4302 (2014). doi: 10.19026/rjaset.7.801

Lukman, R., and Glavič, P. (2007). What are the key elements of a sustainable university? Clean Technol. Environ. Policy. 9, 103-114. doi: $10.1007 / \mathrm{s} 10098-006-0070-7$

Miles, M. B., and, Huberman, M. A. (1994). Qualitative Data Analysis: An Expanded, Sourcebook. Thousand Oaks, CA: Sage Publications.

Miralles-Guasch, C., Domene, E. (2010). Sustainable transport challenges in a suburban university: The case of the Autonomous University of Barcelona. Transport. Policy. 17, 454-463. doi: 10.1016/j.tranpol.2010.04.012

Nejati, M. and Nejati, M. (2013). Assessment of sustainable university factors from the perspective of university students. J. Clean. Prod. 48, 101-107. doi: 10.1016/j.jclepro.2012.09.006

Nieuwenhuijsen, M. J. (2020). Urban and transport planning pathways to carbon neutral, liveable and healthy cities; A review of the current evidence. Environ. Int. 140, 105661. doi: 10.1016/j.envint.2020.105661

Nolan, D. P., and Anderson, E. T. (2015). Applied Operational Excellence for the Oil, Gas, and Process Industries, 1st Edn. New York, NY: Elsevier. p. 266.

OECD/ITF. (2020). Road safety anual report 2020. Available online at: https:// www.itf-oecd.org/sites/default/files/docs/irtad-road-safety-annual-report2020_0.pdf

Papantoniou, P., Vlahogianni, E., Yannis, G., Attard, M., Mora, P. V., Diaz, E. C., et al. (2018). Data Analytics: Paving the Way to Sustainable Urban Mobility: Proceedings of 4th Conference on Sustainable Urban Mobility (CSUM2018). 879, 24-5. doi: 10.1007/978-3-030-02305-8_46

Papantoniou, P., Vlahogianni, E., Yannis, G., Jajac, N., Mimica, M., Andričevi,ć, R., et al. (2017). Deliverable D3.3.2: GAP Analysis, of the research project "CAMPus sustainable University mobility plans in MED areas".

Papantoniou, P., Vlahogianni, E., Yannis, G., Jajac, N., Mimica, M., Andričević, R., et al. (2017). Deliverable D3.3.3: State of the art, of the research project "CAMPus sustainable University mobility plans in MED areas".

Papantoniou, P., Vlahogianni, E., Yannis, G., Papaleo, V., Soluri, D., Jajac, N., et al. (2017). Deliverable D3.2.1: Quantitative data, of the research project "CAMPus sustainable University mobility plans in MED areas").

Papantoniou, P., Vlahogianni, E., Yannis, G., Papaleo, V., Soluri, D., Jajac, N., et al. (2017). Deliverable D3.2.2: Qualitative Information, of the research project "CAMPus sustainable University mobility plans in MED areas".

Papantoniou, P., Vlahogianni, E., Yannis, G., Papaleo, V., Soluri, D., Jajac, N., et al. (2017). Deliverable D3.3.1: SWOT Analysis, of the research project "CAMPus sustainable University mobility plans in MED áreas".

Papantoniou, P., Yannis, G., Vlahogianni, E., Attard, M., Regattieri, A., Piana, F., et al. (2020). Developing a Sustainable Mobility Action Plan for University Campuses. Transpor. Res. Procedia. 48, 1908-1917. doi: 10.1016/j.trpro.2020.08.223

Pitsiava-Latinopoulou, M., Basbas, S., and Gavanas, N. (2013). Implementation of alternative transport networks in university campuses: the case of the Aristotle University of Thessaloniki, Greece. Int. J. Sustain. High. Educ. 14, 310-323 doi: 10.1108/IJSHE-12-2011-0084

Raslavičius, L., Azzopardi, B., Keršys, A., Starevičius, M., Bazaras, Ž., Makaras, R. (2015). Electric vehicles challenges and opportunities: Lithuanian review. Renew. Sust. Energ. Rev. 42, 786-800. doi: 10.1016/j.rser.2014.10.076

Regattieri, A., Pilati, F., Piana, F., Tormo Lancero, M. T., Campos Díaz, E., Valero Mora, P., et al. (2018). Deliverable D3.4.1: Action Plan of sump in urban area. Project CAMP-sUmp sustainable University mobility plans in MED Urban areas".
Regattieri, A., Pilati, F., Piana, F., Tormo Lancero, M. T., Campos Díaz, E., Valero Mora, P., et al. (2018). Deliverable D3.4.2: Action Plan of sump outside urban area. CAMP-sUmp sustainable University mobility plans in MED Urban areas.

Riggs, W. (2020). Telework and sustainable travel during the covid-19 era. Available at SSRN 3638885. doi: 10.2139/ssrn.3638885

Sdoukopoulos, E., Kose, P., Gal-Tzur, A., Mezghani, M., Boile, M., Sheety, E., et al. (2016). Assessment of urban mobility needs, gaps and priorities in Mediterranean partner countries, 6th Transport Research Arena April 18-21, 2016. Transpor. Res. Procedia. 4, 1211-1220 doi: 10.1016/j.trpro.2016.05.192

Silva, J., and Ferreira, D. (2008). European Best Practice on Sustainable Mobility in University Campus, T.aT. - Students Today, Citizens Tomorrow, Report SMILE project. Available online at: http://smile-einfachmobil.at/index_en.html

STARS. The Sustainability Tracking, Assessment and Rating System ${ }^{\text {TM }}$ (STARS). Available online at: https://stars.aashe.org

Thomas, F. M., Charlton, S. G., Lewis, I., and Nandavar, S. (2021). Commuting before and after COVID-19. Transportation Research Interdisciplinary Perspectives, 11, 100423. doi: 10.1016/j.trip.2021.100423

Tolley, R. (1996). Green campuses: cutting the environmental cost of commuting. J. Transport Geography. 4, 213-217. doi: 10.1016/0966-6923(96)000 22-1

Tormo Lancero, M. T., Campos Díaz, E., Valero Mora, P., Papantoniou, P., Vlahogianni, E., Yannis, G., et al. (2018) Deliverable D3. 5. 1 Roadmap for decision makers. "CAMPus sustainable University mobility plans in MED areas".

Torrisi, V., Garau, C., Ignaccolo, M., and Inturri, G. (2020). Sustainable Urban Mobility Plans: Key Concepts and a Critical Revision on SUMPs Guidelines. International Conference on Computational Science and Its Applications. Springer. p. 613-628doi: 10.1007/978-3-030-58820-5_45

Tsirimpa, A., Gkotsis, I., Kepaptsoglou, K., Vlahogianni, E., Polydoropoulou, A., and Karlaftis, M. ${ }^{\dagger}$ (2015). Policies for Enhancing Mobility in Academic Campuses: The case of CERN, Presented to the 7th Conference on Transport Research (ICTR2015), 5-6 November. Athens:

UMOB LIFE. (2017). Available online at: http://u-mob.eu/

University of Bristol. (2017). Combined Travel Plan 2009 - 2016, University of Bristol publications.

Vlahogianni, E., Papantoniou, P., Yannis, G., Attard, M., Regattieri, A., Piana, F., et al. (2018). "Analysis of mobility patterns in selected university campus areas," in Data Analytics: Paving the Way to Sustainable Urban Mobility. CSUM 2018. Advances in Intelligent Systems and Computing, Vol. 879, eds E. Nathanail and I. Karakikes I. (Cham: Springer). doi: 10.1007/978-3-030-023058_52

Wefering, F., Rupprecht, S., Bührmann, S., and Böhler-Baedeker, S. (2014). Guidelines. Developing and Implementing a Sustainable Urban Mobility Plan. European Platform on Sustainable Urban Mobility Plans, European Commission. Directorate-General for Mobility and Transport.

Zhang, J., Hayashi, Y., and Frank, L. D. (2021). COVID-19 and transport: Findings from a world-wide expert survey. Transport policy. 103, 68-85. doi: 10.1016/j.tranpol.2021.01.011

Conflict of Interest: The authors declare that the research was conducted in the absence of any commercial or financial relationships that could be construed as a potential conflict of interest.

Publisher's Note: All claims expressed in this article are solely those of the authors and do not necessarily represent those of their affiliated organizations, or those of the publisher, the editors and the reviewers. Any product that may be evaluated in this article, or claim that may be made by its manufacturer, is not guaranteed or endorsed by the publisher.

Copyright (c) 2022 Tormo-Lancero, Valero-Mora, Sanmartin, Sánchez-García, Papantoniou, Yannis, Alonso and Campos-Díaz. This is an open-access article distributed under the terms of the Creative Commons Attribution License (CC BY). The use, distribution or reproduction in other forums is permitted, provided the original author(s) and the copyright owner(s) are credited and that the original publication in this journal is cited, in accordance with accepted academic practice. No use, distribution or reproduction is permitted which does not comply with these terms. 